Mathematical Modelling and Analysis

Volume 18 Number 2, April 2013, 274-288

http://dx.doi.org/10.3846/13926292.2013.783885

(c) Vilnius Gediminas Technical University, 2013
Publisher: Taylor\&Francis and VGTU

http://www.tandfonline.com/TMMA

Print ISSN: $1392-6292$

Online ISSN: 1648-3510

\title{
Convergence of the Finite Volume Method for a Conductive-Radiative Heat Transfer Problem*
}

\section{Karlis Birgelis and Uldis Raitums}

\author{
Faculty of Physics and Mathematics, University of Latvia \\ Zellu st. 8, LV-1002 Riga, Latvia \\ E-mail: k.birgelis@gmail.com
}

Received September 15, 2012; revised March 5, 2013; published online April 1, 2013

\begin{abstract}
We show that the finite volume method rigorously converges to the solution of a conductive-radiative heat transfer problem with nonlocal and nonlinear boundary conditions. To get this result, we start by proving existence of solutions for a finite volume discretization of the original problem. Then, by obtaining uniform boundedness of discrete solutions and their discrete gradients with respect to mesh size, we finally get $L_{2}$ type convergence of discrete solutions.

Keywords: conductive-radiative heat transfer, finite volume method, nonlocal and nonlinear boundary conditions, convergence.
\end{abstract}

AMS Subject Classification: 35J65.

\section{Introduction}

The main interest of the paper is the applicability of the finite volume method to problems with nonlocal and nonlinear boundary conditions for a linear elliptic equation of the divergence type. As a rule, given class of boundary value problems arise in mathematical modeling of conductive-radiative heat transfer, see, for instance, $[1,2,3,4,8,9]$.

The mathematical model, considered in this paper, involves a rectangular parallelepiped $\Omega$ as a problem domain, a simple convection-diffusion equation and nonlocal nonlinear boundary conditions of the type

$$
\frac{\partial u}{\partial n}(x)+\kappa\left\{|u(x)|^{3} u(x)-H\left[|u(\cdot)|^{3} u(\cdot)\right]\right\}=g(x), \quad x \in \Gamma,
$$

on some part of the boundary $\Gamma \subset \partial \Omega$ (precise formulation of the problem is given in Section 2). Here $H \in \mathcal{L}\left(L_{5 / 4}(\Gamma) \rightarrow L_{5 / 4}(\Gamma)\right)$ is a nonlocal contraction operator of the following type:

$[H(v)](x)=\left[\left((1-\epsilon) I+\epsilon K(I-(1-\epsilon) K)^{-1} \epsilon\right)\left(\begin{array}{l}v \\ 0\end{array}\right)\right](x), v \in L_{5 / 4}(\Gamma), x \in \Gamma$,

* This work was supported by the Latvian Council of Sciences under grant 09.1572 and by the ESF project No. 2009/0223/IDP/1.1.1.2.0/APIA/008. 
where $\epsilon(x) \in(0,1]$ is a function defined on $\Gamma \cup \Gamma_{h}\left(\Gamma_{h}\right.$ is some regular surface), $I$ is the identity operator and $K \in \mathcal{L}\left(L_{5 / 4}\left(\Gamma \cup \Gamma_{h}\right) \rightarrow L_{5 / 4}\left(\Gamma \cup \Gamma_{h}\right)\right)$ is an integral operator of type

$$
\begin{aligned}
& {\left[K\left(\begin{array}{l}
v_{1} \\
v_{2}
\end{array}\right)\right]\left(\begin{array}{l}
x \\
y
\end{array}\right)=\left(\begin{array}{l}
\int_{\Gamma} k(x, t) v_{1}(t) d S+\int_{\Gamma_{h}} k(x, z) v_{2}(z) d S \\
\int_{\Gamma} k(y, t) v_{1}(t) d S+\int_{\Gamma_{h}} k(y, z) v_{2}(z) d S
\end{array}\right),} \\
& v_{1} \in L_{5 / 4}(\Gamma), v_{2} \in L_{5 / 4}\left(\Gamma_{h}\right), x, t \in \Gamma, y, z \in \Gamma_{h},
\end{aligned}
$$

where for arbitrary points $r, s \in \Gamma \cup \Gamma_{h}$

$$
k(r, s)=\frac{\cos (n(r),(s-r)) \cos (n(s),(r-s))}{\pi|r-s|^{2}} \theta(r, s) .
$$

Here $\theta(r, s) \in\{0 ; 1\}$ denotes visibility factor, $n(r), n(s)$ are normal unit vectors to $\Gamma \cup \Gamma_{h}$.

A question about convergence of finite volume schemes is widely discussed in literature. In $[6,7]$ authors give complete framework for convergence analysis of finite volume schemes applied for discretization of linear conductive-convective problems with general boundary conditions. Even convergence analysis for pure radiative, or conductive-convective problems with local Stefan-Boltzmann type boundary conditions can be found (see [5]).

As it turns out, the framework provided by $[6,7]$ can not be directly applied to the boundary value problems having boundary conditions of type (1.1), since convergence analysis for the problems having Neumann or Robin type boundary conditions is heavily based on assumption about at least $W_{2}^{2}$ regularity of solutions of the problem. In our case it is still not clear, if solutions of the boundary value problem involving boundary conditions of type (1.1) have $W_{2}^{2}(\Omega)$ regularity.

Nevertheless, to perform convergence analysis, we took the framework provided by $[6,7]$ and adapted it for our case, when we expect only $W_{2}^{1}(\Omega) \cap L_{\infty}(\Omega)$ type regularity for weak solutions of the boundary value problem.

Whereas standard finite volume schemes give weak convergence of discretized gradients and, as a consequence, strong convergence in spaces $L_{p}(\Omega)$, the nonlinearity in (1.1) demands strong convergence of an equivalent of traces on $\partial \Omega$ of solutions of discretized equations. Besides that, the non-locality of the operator $H$ demands specific methods to obtain uniform (with respect to meshes) a priori estimates for solutions of discretized equations.

For continuous case these difficulties are tackled via embedding theorems in Sobolev spaces, see, for instance [9], or specific trial functions, see, for instance $[1,2,3]$, which unlikely have discrete analogues.

Both problems for cases with rectangular geometry can be bypassed within the framework of the finite volume method, especially to establish invertibility of linearized operators for the discrete case.

The paper is organized as follows. In Section 2 we give the accurate description of the problem and main assumptions on data. After that we show how to reduce the original problem to a more convenient one with linear growth at infinity of the nonlinearity in Stefan-Boltzmann law. In Section 3 we show that the linearized operators are uniformly invertible (for a fixed mesh), what 
together with their Lipschitz continuity give unique solvability of discrete equations. Sections 4-6 are devoted to uniform estimates (independent from the mesh) for discrete gradients and the proof that solutions of discrete equations converge strongly in $L_{2}(\Omega)$ and $L_{2}(\partial \Omega)$ (as the size of meshes goes to zero) to the solution of the initial continuous problem.

\section{Preliminaries}

Let the problem domain $\Omega \subset \mathbb{R}^{3}$ be rectangular parallelepiped $\Omega:=\left(0, a_{1}\right) \times$ $\left(0, a_{2}\right) \times\left(0, a_{3}\right)$ with boundary $\partial \Omega=\Gamma_{0} \cup \Gamma_{1} \cup \Gamma, \Gamma_{0}$ being the intersection of $\bar{\Omega}$ with the plane $\left\{x_{3}=0\right\}, \Gamma_{1}$ being the intersection of $\bar{\Omega}$ with the plane $\left\{x_{3}=a_{3}\right\}$ and $\Gamma$ being the lateral boundary of $\Omega$.

Let us consider the following conductive-radiative heat transfer problem

$$
\left\{\begin{array}{l}
-\triangle u+\gamma u_{x_{3}}=0, \quad x \in \Omega, \\
\left.u\right|_{\Gamma_{0}}=u_{*},\left.\quad \frac{\partial u}{\partial n}\right|_{\Gamma_{1}}=0, \\
\frac{\partial u}{\partial n}+\left.\kappa\left\{|u|^{3} u-H\left[|u(\cdot)|^{3} u(\cdot)\right]\right\}\right|_{\Gamma}=g
\end{array}\right.
$$

and suppose that the following assumptions hold on data:

$H_{1} . \gamma>0, \kappa>0, g \in L_{\infty}(\Gamma), u_{*} \in C^{2}\left(\mathbb{R}^{3}\right)$ and $u_{*}$ does not depend on $x_{3}$. Moreover, we suppose that $0 \leq g(x) \leq d_{0}$ a.e. on $x \in \Gamma$ and $0 \leq u_{*}(x) \leq d_{0}$ a.e. on $x \in \Gamma_{0}$ for some constant $d_{0}$;

It turns out, that under appropriate restrictions on geometry of $\Gamma_{h}$, the nonlocal operator $H$ will have the following properties (see, for instance $[2,8]$ ):

$H_{2} . H \in \mathcal{L}\left(L_{p}(\Gamma) \rightarrow L_{p}(\Gamma)\right),\|H\| \leq q<1$ for all $1 \leq p \leq \infty$ and $H$ maps the nonnegative cone of $L_{p}(\Gamma)$ into itself, $1 \leq p \leq \infty$.

Furthermore, if $H_{1}, H_{2}$ hold then for the problem (2.1) there exists one and only one weak solution $u$ in a Sobolev type space $\left\{v \in W_{2}^{1}(\Omega):\left.v\right|_{\Gamma} \in L_{5}(\Gamma)\right\}$ and $0 \leq u(x) \leq d_{1}$ a.e $x \in \Omega$, where $d_{1}:=\max \left\{\left(\frac{d_{0}}{\kappa(1-q)}\right)^{1 / 4}, d_{0}\right\}$ (see, for instance $[2,8])$. Therefore, by taking this into account, we can replace the original problem with the equivalent one:

$$
\left\{\begin{array}{l}
-\triangle u+\gamma u_{x_{3}}=0, \quad x \in \Omega, \\
\left.u\right|_{\Gamma_{0}}=u_{*},\left.\quad \frac{\partial u}{\partial n}\right|_{\Gamma_{1}}=0, \\
\frac{\partial u}{\partial n}+\left.\kappa\{\psi(u)-H[\psi(u(\cdot))]\}\right|_{\Gamma}=g,
\end{array}\right.
$$

where original Stefan-Boltzmann type nonlinearity $|t|^{3} t$ is replaced with a new one $\psi(t)$, which coincide with Stefan-Boltzmann type nonlinearity only on interval $\left[-d_{1}, d_{1}\right]$, but at infinity has linear growth rate:

$$
\psi(t):= \begin{cases}|t|^{3} t, & |t| \leq d_{1}, \\ 4 d_{1}^{3} t-3 d_{1}^{4}, & t>d_{1}, \\ 4 d_{1}^{3} t+3 d_{1}^{4}, & t<-d_{1} .\end{cases}
$$


It is easy to verify, that: that

$H_{3} . \quad \psi \in C^{1,1}(\mathbb{R})$ and there exist some constants $\mu>0, L \geq 0, \nu>0$ such

$$
\begin{aligned}
& 0 \leq \psi^{\prime}(t) \leq \mu, \quad t \in \mathbb{R} \\
& \left|\psi^{\prime}\left(t_{1}\right)-\psi^{\prime}\left(t_{2}\right)\right| \leq L\left|t_{1}-t_{2}\right|, \quad t_{1} \in \mathbb{R}, t_{2} \in \mathbb{R} \\
& \psi(t) t \geq \nu t^{2}, \quad|t| \geq d_{1}
\end{aligned}
$$

Let $\mathcal{M}$ denote a regular structured mesh on $\Omega$ (see, for instance, $[6,7]$ ) with rectangular cells of type $K=\left(a_{1 K}, b_{1 K}\right) \times\left(a_{2 K}, b_{2 K}\right) \times\left(a_{3 K}, b_{3 K}\right) \subset \mathbb{R}^{3}$. Let us assume, that $\mathcal{M}$ satisfies the following regularity condition: there exists there exists a positive constant $c_{0}$ such that

$$
c_{0}^{-1}(\operatorname{diam} K)^{3} \leq|K| \leq c_{0}(\operatorname{diam} K)^{3} \quad \forall K \in \mathcal{M} .
$$

Here by $|K|$ we denote the Lebesgue measure of $K$ and

$$
\operatorname{diam} K:=\sup _{x, y \in K}|x-y| .
$$

Let us also define the following functional space:

$$
L(\mathcal{M}):=\left\{v \in L_{2}(\Omega) \mid v \text { is constant in every } K \in \mathcal{M}\right\} .
$$

In what follows we will use the following notations (for $v \in L(\mathcal{M})$ ): $d_{\mathcal{M}}$ - the size of the mesh $\mathcal{M}\left(d_{\mathcal{M}}:=\max _{K \in \mathcal{M}}\right.$ diam $\left.K\right) ; \sigma$ - one of six faces (façades) of a cell $K ; \mathcal{E}_{K}$ - the collection of all six faces of a cell $K ; \mathcal{E}$ - the union of all faces $\sigma \in \mathcal{E}_{K}, K \in \mathcal{M} ; K \mid L$ - the face $\sigma$ that is common to $K$ and $L ; \bar{n}_{K \sigma}$ - the unit normal to $\sigma \in \mathcal{E}_{K}$ outward to $K ; \bar{n}$ - unit normal to $\partial \Omega$ outward to $\Omega ; \bar{e}_{i}-$ unit vector aligned in direction of the axis of co-ordinates $O x_{i}, i=1,2,3 ; x_{K}$ - the barycenter of $K ; h_{K \sigma}$ - size of $K$ in the direction orthogonal to $\sigma \in \mathcal{E}_{K}$; $|\sigma|$ - two-dimensional measure of $\sigma \in \mathcal{E} ; v_{K}$ - value of $v$ in the cell $K$. For $\sigma=K \mid L$ let us define:

$$
v_{\sigma}:=\frac{h_{L \sigma} v_{K}+h_{K \sigma} v_{L}}{h_{K \sigma}+h_{L \sigma}} .
$$

For those $\sigma \in \mathcal{E}_{K}$ that belong to $\partial \Omega$ we introduce "artificial" cells $\hat{K}$ as mirror reflections of $K$ over the hyperplane, to which belongs the corresponding $\sigma$. The corresponding values $\hat{v}_{K}=v_{L}$ for $L=\hat{K}$ are defined specifically in accordance to boundary conditions on the corresponding part of $\partial \Omega$ and then

$$
v_{\sigma}:=\frac{\left(v_{K}+\hat{v}_{K}\right)}{2} .
$$

In what follows we will also use the following notations: $\operatorname{tr} v-$ for an element $v$ the trace $\operatorname{tr} v$ as an element of $L_{p}(\partial \Omega), 1 \leq p \leq \infty$ is defined as

$$
\operatorname{tr} v(\cdot):=\sum_{K \in \mathcal{M}} \sum_{\sigma \in \mathcal{E}_{K} \& \sigma \subset \partial \Omega} \chi_{\sigma}(\cdot) v_{\sigma},
$$


where $\chi_{\sigma}$ is the characteristic function of $\sigma \subset \partial \Omega ; \bar{\nabla} v$ - for an element $v$ the discrete gradient $\bar{\nabla} v \in(L(\mathcal{M}))^{3}$ is defined as

$$
\bar{\nabla} v:=\frac{1}{|K|} \sum_{\sigma \in \mathcal{E}_{K}}|\sigma| \bar{n}_{K \sigma}\left(v_{\sigma}-v_{K}\right) \quad \text { in } K, K \in \mathcal{M}
$$

$\sigma_{K+}$ and $\sigma_{K-}$ - faces of $K \in \mathcal{M}$ that are orthogonal to the axis $O x_{3}$ of coordinates, where $\sigma_{K+}$ corresponds to that face, points of which have greater values of $x_{3}$ than points of $\sigma_{K-} ; K_{+}$and $K_{-}-$cells that have with $K$ common face $\sigma_{K+}$ and $\sigma_{K-}$ respectively; $v_{K+}$ and $v_{K-}$ - values of $v$ on the cells $K_{+}$ and $K_{-}$respectively; $l_{\sigma}$ - for a function $l \in L_{1}(\sigma)$ and $\sigma \in \mathcal{E}, \sigma \subset \partial \Omega$

$$
l_{\sigma}:=\frac{1}{|\sigma|} \int_{\sigma} l(x) d S .
$$

Here, if necessary to accent to which $K$ the face $\sigma$ corresponds, we write $l_{K \sigma}$.

In accordance to boundary conditions in (2.2) for a cell $K \in \mathcal{M}$ with a face $\sigma \in \mathcal{E}_{K}$ and $\sigma \subset \partial \Omega$, we set (for $v \in L(\mathcal{M})$ )

$$
\begin{aligned}
\hat{v}_{K} & :=v_{K}, \quad \text { if } \sigma \in \mathcal{E}_{K} \text { and } \sigma \subset \Gamma_{1} \\
\hat{v}_{K} & :=2 u_{* \sigma}-v_{K}, \quad \text { if } \sigma \in \mathcal{E}_{K} \text { and } \sigma \subset \Gamma_{0} \\
\hat{v}_{K} & :=v_{K}-h_{K \sigma} G(v)_{K \sigma}, \quad \text { if } \sigma \in \mathcal{E}_{K} \text { and } \sigma \subset \Gamma ; \\
G(v)_{K \sigma} & :=\kappa\left\{\frac{1}{|\sigma|} \int_{\sigma} \psi\left(v_{K}\right) d S-\frac{1}{|\sigma|} \int_{\sigma} H[\psi(v)](x) d S\right\}-\frac{1}{|\sigma|} \int_{\sigma} g d S .
\end{aligned}
$$

Here the argument of the operator $H$ is understood as $\psi(v):=\psi\left(v_{K}\right)$, if $x \in$ $\Gamma \cap \bar{K}$.

Now, by integrating the elliptic equation of (2.2) over each cell $K \in \mathcal{M}$ and approximating resulting diffusive and convective fluxes with standard central and upwind schemes, respectively (see $[6,7]$ ), we will obtain the family of equations

$$
F_{K}(w):=-\sum_{\sigma \in \mathcal{E}_{K}} 2 \frac{|\sigma|}{h_{\sigma}}\left(w_{\sigma}-w_{K}\right)+\gamma\left(\left|\sigma_{K+}\right| w_{K}-\left|\sigma_{K-}\right| w_{K-}\right)=0, \quad K \in \mathcal{M},
$$

where $w \in L(\mathcal{M})$ is approximate solution of $u$ and for each cell that have a face on boundary $\partial \Omega$ the formulas (2.3) are used to calculate $w_{\sigma}$.

The family of equations (2.4) we will denote shortly as

$$
F(w)-\bar{f}=0,
$$

where $F: L(\mathcal{M}) \rightarrow L(\mathcal{M})$ and $\bar{f}$ incorporate the corresponding values of $u_{* \sigma}$ and $g_{\sigma}$, which come from (2.3) for $w_{\sigma}$ with $\sigma \in \mathcal{E}_{K}$ and $\sigma \subset \partial \Omega$.

Along with the equation $F(w)-\bar{f}=0$ we will consider the linearized equation

$$
\begin{gathered}
F^{\prime}(\alpha) \tilde{w}=\tilde{f}, \\
F^{\prime}(\alpha): L(\mathcal{M}) \rightarrow L(\mathcal{M}),
\end{gathered}
$$


with $\alpha \in L_{\infty}(\Gamma), 0 \leq \alpha(x) \leq \mu$ a.e. $x \in \Gamma$, where

$$
\begin{aligned}
F_{K}^{\prime}(\alpha) \tilde{w} & :=\left.F^{\prime}(\alpha) \tilde{w}\right|_{K} \\
& :=-\sum_{\sigma \in \mathcal{E}_{K}} 2 \frac{|\sigma|}{h_{\sigma}}\left(\tilde{w}_{\sigma}-\tilde{w}_{K}\right)+\gamma\left(\left|\sigma_{K}\right| \tilde{w}_{K+}-\left|\sigma_{K-}\right| \tilde{w}_{K-}\right), \quad K \in \mathcal{M},
\end{aligned}
$$

and for $\sigma \in \mathcal{E}_{K}$ and $\sigma \subset \partial \Omega$ the corresponding values of $\hat{\tilde{w}}$ for $\sigma$ are defined by analogy to formulas (2.3):

$$
\begin{aligned}
\hat{\tilde{w}}_{K} & :=\tilde{w}_{K}, \quad \text { if } \sigma \subset \Gamma_{1}, \quad \hat{\tilde{w}}_{K}:=-\tilde{w}_{K}, \quad \text { if } \sigma \subset \Gamma_{0}, \\
\hat{\tilde{w}}_{K} & :=\tilde{w}_{K}-h_{K \sigma} G^{\prime}(\alpha \tilde{w})_{K \sigma}, \quad \text { if } \sigma \subset \Gamma, \\
G^{\prime}(\alpha \tilde{w})_{K \sigma} & :=\kappa\left\{\frac{1}{|\sigma|} \int_{\sigma} \alpha(x) \tilde{w}_{K} d S-\frac{1}{|\sigma|} \int_{\sigma} H[\alpha(\cdot) \tilde{w}](x) d S\right\}, \\
\alpha(\cdot) \tilde{w} & :=\alpha(x) \tilde{w}_{K}, \quad \text { if } x \in \Gamma \cap \bar{K} .
\end{aligned}
$$

\section{Solvability of the Discretized Equation}

We start with the following lemma.

Lemma 1. Let the hypotheses $H_{1}, H_{2}, H_{3}$ hold and let $\alpha \in L_{\infty}(\Gamma)$ be nonnegative. Then the homogeneous linearized equation

$$
F^{\prime}(\alpha) \tilde{w}=0
$$

with respect to $\tilde{w} \in L(\mathcal{M})$ has only trivial solution.

Proof. Let us multiply expressions (2.5) for the cell $K$ by $\operatorname{sign} \tilde{w}_{K}$ and sum up. We have

$$
\begin{aligned}
0= & -\sum_{K \in \mathcal{M}} \sum_{\sigma \in \mathcal{E}_{K}} 2 \frac{|\sigma|}{h_{\sigma}}\left(\tilde{w}_{\sigma}-\tilde{w}_{K}\right) \operatorname{sign} \tilde{w}_{K} \\
& +\gamma \sum_{K \in \mathcal{M}}\left(\left|\sigma_{K+}\right| \tilde{w}_{K}-\left|\sigma_{K-}\right| \tilde{w}_{K-}\right) \operatorname{sign} \tilde{w}_{K} \\
= & \sum_{\sigma \in \mathcal{E} \& \sigma \not \subset \partial \Omega} \frac{2|\sigma|}{h_{K \sigma}+h_{L \sigma}}\left|\tilde{w}_{L}-\tilde{w}_{K}\right| \cdot\left|\operatorname{sign} \tilde{w}_{L}-\operatorname{sign} \tilde{w}_{K}\right| \\
& +\sum_{K \in \mathcal{M}} \sum_{\sigma \in \mathcal{E}_{K} \& \sigma \subset \Gamma_{0}} \frac{|\sigma|}{h_{\sigma}} \tilde{w}_{K} \operatorname{sign} \tilde{w}_{K} \\
& +\sum_{K \in \mathcal{M}} \sum_{\sigma \in \mathcal{E} \& \sigma \subset \Gamma}|\sigma| G^{\prime}(\alpha \tilde{w})_{K \sigma} \operatorname{sign} \tilde{w}_{K} \\
& +\gamma \sum_{K \in \mathcal{M}}\left|\sigma_{K+}\right| \cdot\left|\tilde{w}_{K}\right| \cdot\left|\operatorname{sign} \tilde{w}_{K}-\operatorname{sign} \tilde{w}_{K+}\right| \\
& +\gamma \sum_{K \in \mathcal{M} \& \sigma_{K-} \subset \Gamma_{0}}\left|\sigma_{K-}\right| \cdot\left|\tilde{w}_{K}\right| .
\end{aligned}
$$


From here it follows

$$
\kappa \int_{\Gamma} G^{\prime}(\alpha \tilde{w}) \operatorname{sign} \tilde{w} d S=\kappa \int_{\Gamma} \alpha|\operatorname{tr} \tilde{w}| d S-\kappa \int_{\Gamma} H[\alpha \tilde{w}] \operatorname{sign} \operatorname{tr} \tilde{w} d S \leq 0 .
$$

Since $\alpha(\cdot) \geq 0$ then from hypothesis $H_{2}$ it follows

$$
\|\alpha \tilde{w}\|_{L_{\infty}} \leq q\|\alpha \tilde{w}\|_{L_{\infty}},
$$

i.e. $\alpha \tilde{w}=0$ on $\Gamma$. After that, from (3.1) it follows immediately that $\tilde{w} \equiv 0$.

Corollary 1. For any nonnegative $\alpha \in L_{\infty}(\Gamma)$ the operator $F^{\prime}(\alpha)$ is invertible and there exists a constant $c_{1}=c_{1}(\mathcal{M}, \mu)$ such that for all $\alpha \in L_{\infty}(\Gamma), 0 \leq$ $\alpha(x) \leq \mu, x \in \Gamma$, there is

$$
\left\|\left[F^{\prime}(\alpha)\right]^{-1}\right\| \leq c_{1}
$$

Proof. The proof immediately follows from the fact that the whole family $\left\{F^{\prime}(\alpha)\right\}$ forms a compact set in $\mathcal{L}(L(\mathcal{M}) \rightarrow L(\mathcal{M}))$.

Remark 1. Here and in what follows it is supposed that constants in hypotheses $H_{1}, H_{2}$ and $H_{3}$, and also the constant $c_{0}$ are fixed once and for all.

Theorem 1. For every fixed regular mesh $\mathcal{M}$ and every fixed $\bar{f} \in L(\mathcal{M})(\bar{f}$ is defined by $\left.u_{*} \in C^{2}\left(\mathbb{R}^{2}\right), g \in L_{\infty}(\Gamma)\right)$ the equation $F(w)=\bar{f}$ is uniquely solvable in $L(\mathcal{M})$.

Proof. By virtue of hypotheses $H_{1}, H_{2}$ and $H_{3}$ the operator $F$ is Fréchet differentiable and its Fréchet derivative on an element $w \in L(\mathcal{M})$ has the representation

$$
F^{\prime}(w)=F^{\prime}(\alpha), \quad \alpha(x):=\psi^{\prime}\left(w_{K}\right), \quad x \in \Gamma \cap \bar{K},
$$

with $0 \leq \alpha(x) \leq \mu$. Further, again by virtue of $H_{2}$ and $H_{3}$ the mapping

$$
L(\mathcal{M}) \ni w \rightarrow F^{\prime}(w) \in \mathcal{L}(L(\mathcal{M}) \rightarrow L(\mathcal{M}))
$$

is uniformly Lipschitz continuous and one can apply various damped Newton methods (see, for instance, [3] for the continuous case) to obtain unique solvability of the equation $F(w)=\bar{f}$.

\section{Boundedness of Solutions}

To proceed further, we will need uniform (with respect to regular meshes $\mathcal{M}$ ) a priori estimates for solutions of (2.4).

Theorem 2. Let the hypotheses $H_{1}-H_{3}$ hold. Then there exists a constant $c_{2}$ such that for all regular meshes $\mathcal{M}$ solutions $w$ of (2.4) satisfy

$$
\|w\|_{L_{\infty}(\Omega)} \leq c_{2}, \quad\|\operatorname{tr} w\|_{L_{\infty}(\Gamma)} \leq c_{2} .
$$


Proof. From the maximum principle it follows immediately that a solution $w$ of (2.4) can not achieve its strong local maximum on a cell $K$, which has no common faces with $\partial \Omega$. Therefore, it remains to investigate cases where $w$ attains its positive maximum $a_{+}$on a cell $K$, which has a face $\sigma \subset \partial \Omega$.

If $a_{+} \leq\left\|u_{*}\right\|_{L_{\infty}\left(\Gamma_{0}\right)}$, then we have our estimate.

If $a_{+}>\left\|u_{*}\right\|_{L_{\infty}\left(\Gamma_{0}\right)}$ and $K$ has only one face $\sigma \subset \Gamma_{1}$, then from (2.3) and (2.4) it follows that $w$ attains the same value $a_{+}$on neighborhoods of $K$. Hence, it remains to investigate the case where $K$ has a face $\sigma \subset \Gamma$.

If $a_{+} \leq d_{1}$, then we have our estimate.

If $a_{+}>d_{1}$, then from (2.3) and (2.4) it follows

$$
G(w)_{K \sigma}:=\kappa\left\{\frac{1}{|\sigma|} \int_{\sigma} \psi\left(w_{K}\right) d S-\frac{1}{|\sigma|} \int_{\sigma} H[\psi(w)] d S\right\}-\frac{1}{|\sigma|} \int_{\sigma} g d S \leq 0 .
$$

For this case from $H_{3}$ it it follows that for all cells $L$, which have a common face with $\Gamma$,

$$
\psi\left(w_{L}\right) \leq \psi\left(w_{K}\right) .
$$

From here, (4.1) and hypotheses $H_{2}$ and $H_{3}$ now it follows

$$
\int_{\sigma} \psi\left(w_{K}\right) d S \leq \frac{|\sigma|}{\kappa(1-q)}\|g\|_{L_{\infty}} \leq d_{1}^{4}|\sigma| \text {. }
$$

Therefore, since $\psi\left(a_{+}\right)=4 d_{1}^{3} a_{+}-3 d_{1}^{4}$ for $a_{+}>d_{1}$, then the last inequality will yield $a_{+} \leq d_{1}$, what contradicts with the starting assumption.

Therefore, finally we will have $a_{+} \leq d_{1}$. The case of negative minimum $a_{-}$of $w$ is treated analogously. Moreover. it is possible to show that $a_{-} \geq 0$. Obviously, these estimates for $w$ imply the statement of the theorem.

We point out here that the assertion of Theorem 2 holds true uniformly with respect to all regular meshes $\mathcal{M}$.

\section{Discrete Gradients and Their Convergence as $d_{\mathcal{M}} \rightarrow 0$}

To proceed further, we want to avoid nonzero boundary terms on $\Gamma_{0}$. To this end, we define $\bar{u} \in L(\mathcal{M})$ as

$$
\bar{u}_{K}:=\frac{1}{|K|} \int_{K} u_{*} d x, \quad K \in \mathcal{M}
$$

(for mirror reflections $L$ of cells $K$ with common face $\sigma \subset \partial \Omega$ we use the values of $u_{*}$ outside $\Omega$ ). By virtue of $H_{1}$ the function $\bar{u}$ as an element of $L_{2}(\Omega)$ does not depend on $x_{3}$.

Then the function $v \in L(\mathcal{M}), v:=u-\bar{u}$, will satisfy

$$
\begin{gathered}
-\sum_{\sigma \in \mathcal{E}_{K}} 2 \frac{|\sigma|}{h_{K \sigma}}\left(v_{\sigma}-v_{K}\right)+\gamma\left(\left|\sigma_{K+}\right| v_{K}-\left|\sigma_{K-}\right| v_{K-}\right)+\hat{f}_{K}=0, \\
\hat{f}_{K}:=-\sum_{\sigma \in \mathcal{E}_{K}} 2 \frac{|\sigma|}{h_{K \sigma}}\left(\bar{u}_{\sigma}-\bar{u}_{K}\right), \quad K \in \mathcal{M}
\end{gathered}
$$


and for mirror reflections $L$ of cells $K$ with some $\sigma \subset \partial \Omega$

$$
\begin{aligned}
& v_{L}:=\hat{v}_{K}:=-v_{K}, \quad \text { if } \sigma \subset \Gamma_{0}, \\
& v_{L}:=\hat{v}_{K}:=v_{K}, \quad \text { if } \sigma \subset \Gamma_{1}, \\
& v_{L}:=\hat{v}_{K}:=v_{K}-h_{K \sigma} G(v+\bar{u})_{K \sigma}+\bar{u}_{K}-\hat{\bar{u}}_{K}, \quad \text { if } \sigma \subset \Gamma .
\end{aligned}
$$

Since $u, \bar{u}$ are bounded uniformly with respect to a regular mesh $\mathcal{M}$, then also values of $v \in L(\mathcal{M})$ and values of $\operatorname{tr} v, G(v+\bar{u})$ are bounded by some constant $c_{3}$, which does not depend on the choice of a regular mesh $\mathcal{M}$.

According to our notations, the discrete gradient $\bar{\nabla} v \in L(\mathcal{M})^{3}$ of $v$ is defined as

$$
\bar{\nabla} v(x):=\bar{\nabla} v_{K}:=\frac{1}{|K|} \sum_{\sigma \in \mathcal{E}_{K}}|\sigma| \bar{n}_{K \sigma}\left(v_{\sigma}-v_{K}\right), \quad x \in K, K \in \mathcal{M}
$$

By construction,

$$
\begin{gathered}
\frac{1}{2} \int_{\Omega}|\bar{\nabla} v|^{2} d x \leq \sum_{K \in \mathcal{M}} \sum_{\sigma \in \mathcal{E}_{K} \& \sigma=K \mid L} \frac{|\sigma|}{h_{K \sigma}+h_{L \sigma}}\left|v_{K}-v_{L}\right|^{2}:=\|v\|_{1, \mathcal{M}}^{2}, \\
\|(\zeta v)\|_{1, \mathcal{M}}^{2} \leq 2\|\zeta\|_{C^{1}}^{2} \cdot\|v\|_{1, \mathcal{M}}^{2}+2\left(1+d_{\mathcal{M}}\right)^{2} c_{3}^{2} 6|\Omega| \cdot\|\zeta\|_{C^{1}}^{2}
\end{gathered}
$$

for $\zeta \in C^{1}\left(\mathbb{R}^{3}\right)$, where values of $v_{L}:=\hat{v}_{K}$ for mirror reflections $L$ of $K$ over $\sigma$ are defined according to $(5.2)$ and $\zeta_{K}$ are defined as mean values of $\zeta$ over $K$.

Lemma 2. If $v \in L(\mathcal{M})$ satisfies equations (5.1)-(5.2), then there exists a constant $c_{4}=c_{4}\left(\Omega, d_{1}, q, c_{0}, \kappa, \mu, \nu\right)$ such that for all regular meshes $\mathcal{M}$

$$
\|v\|_{1, \mathcal{M}} \leq c_{4}
$$

Proof. The proof is standard: we multiply equations in (5.1) by corresponding values $v_{K}$ and sum up. That gives

$$
\begin{aligned}
0= & -\sum_{K \in \mathcal{M}} \sum_{\sigma \in \mathcal{E}_{K}} 2 \frac{|\sigma|}{h_{K \sigma}}\left(v_{\sigma}-v_{K}\right) v_{K} \\
& +\gamma \sum_{K \in \mathcal{M}} \sum_{\sigma \in \mathcal{E}_{K}}\left(\left|\sigma_{K+}\right| v_{K}-\left|\sigma_{K-}\right| v_{K-}\right) v_{K}+\int_{\Omega} v \hat{f} d x \\
= & \|v\|_{1, \mathcal{M}}+\sum_{K \in \mathcal{M}} \sum_{\sigma \in \mathcal{E}_{K} \& \sigma \subset \Gamma} \frac{|\sigma|}{2 h_{K \sigma}}\left(v_{K}-\hat{v}_{K}\right) \hat{v}_{K} \\
& +\gamma \sum_{K \in \mathcal{M}} \sum_{\sigma \in \mathcal{E}_{K}}\left|\sigma_{K+}\right|\left(v_{K}-v_{K-}\right) v_{K}+\int_{\Omega} v \hat{f} d x .
\end{aligned}
$$

Since $\hat{v}_{k}=-v_{K}$, if $\sigma \in \Gamma_{0}$ and for an arbitrary vector $\left(a_{1}, \ldots, a_{m}\right)$

$$
\left|\left(a_{1}, \ldots, a_{m}\right)\right|^{2}-\left\langle\left(a_{1}, \ldots, a_{m}\right),\left(-a_{1}, a_{1}, \ldots, a_{m-1}\right)\right\rangle \geq 0
$$

then in the right-hand side of (5.4) the term with the coefficient $\gamma$ is nonnegative. 
Since values of $v_{K}, K \in \mathcal{M}$ (also values $\hat{v}_{K}$ ), are bounded by $c_{3}$, then

$$
\begin{aligned}
\int_{\Omega} \hat{f} v d x= & \sum_{K \in \mathcal{M}} \sum_{\sigma \in \mathcal{E}_{K} \& \sigma=K \mid L} \frac{2|\sigma|}{h_{K \sigma}+h_{L \sigma}}\left(\bar{u}_{K}-\bar{u}_{L}\right)\left(v_{K}-v_{L}\right) \\
& -\sum_{\sigma \subset \partial \Omega} \sum_{\sigma \in \mathcal{E}_{K}} \frac{2|\sigma|}{2 h_{K \sigma}}\left(\hat{\bar{u}}_{K}-\bar{u}_{K}\right) \hat{v}_{K},
\end{aligned}
$$

what gives

$$
\left|\int_{\Omega} \hat{f} v d x\right| \leq 10|\Omega|^{1 / 2} d_{1}\|v\|_{1, \mathcal{M}}+|\Gamma| d_{1} c_{3} .
$$

Finally, due to $(5.2)$,

$$
\begin{aligned}
\frac{|\sigma|}{2 h_{K \sigma}}\left|v_{K}-\hat{v}_{K}\right| & \leq 1 / 2|\sigma| \cdot\left(\left|G(v+\bar{u})_{K \sigma}\right|+d_{1}\right) \leq 1 / 2|\sigma|\left(c_{3}+d_{1}\right) \\
\left|\hat{v}_{K}\right| & \leq\left(c_{3}+d_{1}\right)\left(1+d_{\mathcal{M}}\right) .
\end{aligned}
$$

Therefore

$$
\left|\sum_{K \in \mathcal{M}} \sum_{\sigma \in \mathcal{E}_{K} \& \sigma \subset \Gamma} \frac{|\sigma|}{2 h_{K \sigma}}\left(v_{K}-\hat{v}_{K}\right) \hat{v}_{K}\right| \leq 1 / 2|\Gamma|\left(1+d_{\mathcal{M}}\right)\left(c_{3}+d_{1}\right)^{2} .
$$

From these estimates immediately follows the statement of the lemma.

Now, let $\left\{d_{\mathcal{M}}^{N}\right\}$ be sequence that converges to zero as $N \rightarrow \infty$. To every $N$ we appoint $v_{N}$ as the solution of (5.1)-(5.2) for the mesh $\mathcal{M}^{N}$, which corresponds to $d_{\mathcal{M}}^{N}$.

By virtue of Theorem 2 and Lemma 2, without loss of generality, we can assume that the sequences

$$
\left\{v^{N}\right\} \subset L_{\infty}(\Omega), \quad\left\{\bar{\nabla} v^{N}\right\} \subset L_{2}\left(\Omega ; \mathbb{R}^{3}\right), \quad\left\{\operatorname{tr} v^{N}\right\} \subset L_{\infty}(\partial \Omega)
$$

are uniformly bounded and weak-* converge to

$$
v_{0} \in L_{\infty}(\Omega), \quad \bar{\rho} \in L_{2}\left(\Omega ; \mathbb{R}^{3}\right), \quad \omega \in L_{\infty}(\partial \Omega)
$$

respectively.

We will say that a vector-function $\bar{\eta} \in L_{1}\left(\Omega ; \mathbb{R}^{3}\right)$ is eligible, if the values

$$
\bar{\eta}_{K}:=\frac{1}{|K|} \int_{K} \bar{\eta} d x, \quad \bar{\eta}_{\sigma}:=\frac{1}{|\sigma|} \int_{\sigma}\left\langle\bar{\eta}(\cdot), \bar{n}_{K \sigma}\right\rangle d S \quad \sigma \in \mathcal{E}_{K}, \quad \int_{K} \operatorname{div} \bar{\eta}(x) d x
$$

are defined and finite $(\operatorname{div} \bar{\eta}(\cdot)$ is understood in the sense of distributions) and the relationship

$$
\int_{K} \operatorname{div} \bar{\eta}(x) d x=\sum_{\sigma \in \mathcal{E}_{K}}\left\langle|\sigma| \bar{\eta}_{\sigma}, \bar{n}_{K \sigma}\right\rangle
$$

holds for every $K \in \mathcal{M}$. 
Let $\bar{\eta}$ be an eligible vector-function. We multiply vectors $\bar{\nabla} v_{K}^{N}$ by $|K| \bar{\eta}_{K}$ and sum up. That give

$$
\begin{aligned}
\int_{\Omega}\left\langle\bar{\nabla} v^{N}, \bar{\eta}\right\rangle d x= & \sum_{K \in \mathcal{M}} \sum_{\sigma \in \mathcal{E}_{K}}\left(-v_{K}^{N}\right)\left\langle|\sigma| \bar{\eta}_{\sigma}, \bar{n}_{K \sigma}\right\rangle \\
& +\sum_{K \in \mathcal{M}} \sum_{\sigma \in \mathcal{E}_{K} \& \sigma \subset \partial \Omega} v_{\sigma}^{N}\left\langle|\sigma| \bar{\eta}_{\sigma}, \bar{n}_{K \sigma}\right\rangle+R\left(v^{N}, \bar{\eta}\right),
\end{aligned}
$$

where

$$
\begin{aligned}
R\left(v^{N}, \bar{\eta}\right) & :=\sum_{K \in \mathcal{M}} \sum_{\sigma \in \mathcal{E}_{K}}\left\langle|\sigma| \bar{n}_{K \sigma}, \bar{\eta}_{K}-\bar{\eta}_{\sigma}\right\rangle\left(v_{\sigma}^{N}-v_{K}^{N}\right), \\
\left|R\left(v^{N}, \bar{\eta}\right)\right| & \leq\left\|v^{N}\right\|_{1, \mathcal{M}}\left(\sum_{K \in \mathcal{M}} \sum_{\sigma \in \mathcal{E}_{K} \& \sigma=K \mid L} \frac{h_{\sigma}^{2}}{h_{K \sigma}+h_{L \sigma}}\left\langle\bar{n}_{K \sigma}, \bar{\eta}_{K}-\bar{\eta}_{\sigma}\right\rangle^{2}\right)^{1 / 2},
\end{aligned}
$$

and for every eligible $\bar{\eta}=\nabla \varphi$

$$
\begin{aligned}
& \left|R\left(v^{N}, \nabla \varphi\right)\right| \leq 2 c_{4} \sum_{i=1}^{3}\left(\int_{\Omega}\left(\delta_{i} \varphi(x)\right)^{2} d x\right)^{1 / 2}, \\
& \delta_{i} \varphi(x):=\sup \left\{\left|\varphi_{x_{i}}\left(x+t \bar{e}_{i}\right)-\varphi_{x_{i}}(x)\right|: x+t \bar{e}_{i} \in \Omega,|t| \leq d_{\mathcal{M}}\right\}, \\
& \quad x \in \Omega, i=1,2,3 .
\end{aligned}
$$

By virtue of (5.6), for every $\bar{\eta} \in C^{1}\left(\bar{\Omega} ; \mathbb{R}^{3}\right) R\left(v^{N}, \bar{\eta}\right) \rightarrow 0$ as $N \rightarrow \infty$ and, after passing to the limit $N \rightarrow \infty$ in (5.5),

$$
\int_{\Omega}\langle\bar{\rho}, \bar{\eta}\rangle d x=\int_{\Omega}\left(-v_{0}\right) \operatorname{div} \bar{\eta} d x+\int_{\partial \Omega} \omega\langle\bar{\eta}, \bar{n}\rangle d S,
$$

what gives

$$
v_{0} \in W_{2}^{1}(\Omega), \quad \bar{\rho}=\nabla v_{0}, \quad \omega=\operatorname{tr} v_{0} .
$$

In turn, uniform boundedness of $\left\|v^{N}\right\|_{1, \mathcal{M}},\left\|v^{N}\right\|_{L_{\infty}(\Omega)}$, estimate (5.3) with appropriate cut-off functions $\zeta$ and Lemma 5.5 of [6] give that the sequence $\left\{v^{N}\right\} \subset L_{2}(\Omega)$ is precompact. Therefore, without loosing generality, we can assume that the whole sequence $\left\{v^{N}\right\}$ converges strongly to $v_{0}$ in $L_{2}(\Omega)$ and the whole sequence $\left\{\bar{\nabla} v^{N}\right\}$ converges weakly to $\nabla v_{0}$ in $L_{2}\left(\Omega ; \mathbb{R}^{3}\right)$ as $N \rightarrow \infty$ (we will see later that $v_{0}+u_{*}$ gives the solution of $(2.2)$ and as such is unique).

The space $L_{2}\left(\Omega ; \mathbb{R}^{3}\right)$ is the orthogonal sum

$$
\begin{aligned}
L_{2}\left(\Omega ; \mathbb{R}^{3}\right) & =\left\{\nabla \varphi \mid \varphi \in W_{2}^{1}(\Omega)\right\} \oplus \mathcal{N}, \\
\mathcal{N} & :=\left\{\xi \in L_{2}\left(\Omega ; \mathbb{R}^{3}\right) \mid \operatorname{div} \xi=0,\langle\xi, \bar{n}\rangle=0 \text { on } \partial \Omega\right\} .
\end{aligned}
$$

Hence, $\bar{\nabla} v^{N}$ has the representation

$$
\begin{gathered}
\bar{\nabla} v^{N}=\nabla \varrho^{N}+\xi^{N}, \\
\varrho^{N} \in W_{2}^{1}(\Omega), \quad \xi^{N} \in \mathcal{N}, \quad \varrho^{N} \rightarrow v_{0} \text { weakly in } W_{2}^{1}(\Omega) \text { as } N \rightarrow \infty,
\end{gathered}
$$


and for every $\varphi$, for which $\bar{\eta}=\nabla \varphi$ is eligible,

$$
\begin{aligned}
\int_{\Omega} & \left(-\varrho^{N}\right) \operatorname{div} \nabla \varphi d x+\int_{\partial \Omega} \operatorname{tr} \varrho^{N} \frac{\partial \varphi}{\partial n} d S \\
= & \int_{\Omega}\left\langle\nabla \varrho^{N}, \nabla \varphi\right\rangle d x=\int_{\Omega}\left\langle\bar{\nabla} v^{N}, \nabla \varphi\right\rangle d x=\int_{\Omega}\left(-v^{N}\right) \operatorname{div} \nabla \varphi d x \\
& +\int_{\partial \Omega} \operatorname{tr} v^{N} \frac{\partial \varphi}{\partial n} d S+R\left(v^{N}, \nabla \varphi\right) .
\end{aligned}
$$

Let $\tilde{\sigma} \subset \partial \Omega$ be a subset, which belongs to one face of $\Omega$, and the distance between $\tilde{\sigma}$ and other faces of $\Omega$ is positive. Define $\varphi^{N}$ as

$$
\varphi^{N}(x):=\frac{1}{2 \pi} \int_{\partial \Omega} \chi_{\tilde{\sigma}}(y) \operatorname{tr} v^{N}(y) \frac{1}{|x-y|} d S_{y}, \quad x \in \mathbb{R}^{3} .
$$

Due to Theorem 2 and properties of the Poisson's integrals, see, for instance, I.M. Stein [10, Chapter III], all $\varphi^{N}$ are harmonic in $\Omega$, belong to a bounded set in $C^{2}(\bar{D})$ for every fixed $D \subset \mathbb{R}^{3}$ with $\operatorname{dist}\{\bar{D} ; \tilde{\sigma}\}>0$, and near the hyperplane $E$, to which belongs $\tilde{\sigma}$,

$$
\begin{aligned}
& \left|\frac{\partial \varphi^{N}}{\partial \bar{l}}(x)\right| \leq c_{3}, \quad \text { if } \bar{l} \text { is orthogonal to } E \\
& \left|\frac{\partial \varphi^{N}}{\partial \bar{l}}(x)\right| \leq c_{4}\left(c_{3}, \Omega\right)[1+|\ln (\operatorname{dist}\{x ; E\})|], \quad \text { if } \bar{l} \text { is parallel to } E .
\end{aligned}
$$

These properties are sufficient for that $\bar{\eta}:=\nabla \varphi^{N}$ are eligible and that

$$
R\left(v^{N}, \nabla \varphi^{N}\right) \rightarrow 0 \quad \text { as } N \rightarrow \infty .
$$

Hence, inserting $\nabla \varphi^{N}$ in (5.7) we have

$$
\int_{\tilde{\sigma}}\left(\operatorname{tr} \varrho^{N}-\operatorname{tr} v^{N}\right) \operatorname{tr} v^{N} d S \rightarrow 0 \quad \text { as } N \rightarrow \infty,
$$

and from embedding theorems for $\varrho^{N}$ it follows that the sequence $\left\{\operatorname{tr} v^{N}\right\}$ also converges strongly in $L_{2}(\tilde{\sigma})$ to $\operatorname{tr} v_{0}$.

Arbitrariness of $\tilde{\sigma}$ and the uniform boundedness of $\operatorname{tr} v^{N}$ now imply that the whole sequence $\left\{\operatorname{tr} v^{N}\right\}$ converges to $\operatorname{tr} v_{0}$ strongly in $L_{2}(\partial \Omega)$.

\section{Convergence to the Solution of the Boundary Value Problem}

Obtained in the previous sections properties of convergence for the sequence $\left\{v^{N}\right\}$ are sufficient for that $v^{0}$ gives the solution from $W_{2}^{1}(\Omega)$ of the initial boundary value problem (2.2).

To begin with, we point out that the family of functions $\left\{\bar{u}^{N}\right\}$, where $\bar{u}^{N}$ is defined for the corresponding mesh $\mathcal{M}^{N}$, converges strongly in $L_{2}(\Omega)$ and $L_{2}(\partial \Omega)$ to $u_{*}$. 
Let $\varphi$ be sufficiently smooth function, say, $\varphi \in C^{2}\left(\mathbb{R}^{3}\right)$, which is equal to zero near the boundary $\Gamma_{0}$. Let, for a chosen mesh $\mathcal{M}$,

$$
\varphi_{K}:=\varphi\left(x_{K}\right), \quad \varphi_{\sigma}:=\frac{h_{L \sigma} \varphi_{K}+h_{K \sigma} \varphi_{L}}{h_{L \sigma}+h_{K \sigma}}, \quad \text { if } \sigma=K \mid L, K \in \mathcal{M}, \sigma \in \mathcal{E}_{K},
$$

where $x_{K}$ is the barycenter of $K$ and, if necessary, the mirror reflections over $\sigma \subset \partial \Omega$ are used.

We multiply equations $(5.1)$ by corresponding $\varphi_{K}$ and sum up. We get

$$
\begin{aligned}
& 0=-\sum_{K \in \mathcal{M}} \sum_{\sigma \in \mathcal{E}_{K}} 2 \frac{|\sigma|}{h_{\sigma}}\left(v_{\sigma}-v_{K}\right) \varphi_{K} \\
& +\gamma \sum_{K \in \mathcal{M}}\left(\left|\sigma_{K+}\right| v_{K}-\left|\sigma_{K-}\right| v_{K-}\right) \varphi_{K}+\sum_{K \in \mathcal{M}} \varphi_{K} \hat{f}_{K} \\
& =-\sum_{K \in \mathcal{M}} \sum_{\sigma \in \mathcal{E}_{K}} 2 \frac{|\sigma|}{h_{\sigma}}\left(\varphi_{\sigma}-\varphi_{K}\right) v_{K} \\
& -\gamma \sum_{K \in \mathcal{M}}\left(\left|\sigma_{K+}\right| \varphi_{K}-\left|\sigma_{K-}\right| \varphi_{K-}\right) v_{K} \\
& +\sum_{K \in \mathcal{M}} \sum_{\sigma \in \mathcal{E}_{K} \& \sigma \subset \partial \Omega} 2 \frac{|\sigma|}{h_{\sigma}}\left(\varphi_{\sigma}-\varphi_{K}\right) v_{K} \\
& -\sum_{K \in \mathcal{M}} \sum_{\sigma \in \mathcal{E}_{K} \& \sigma \subset \partial \Omega} \frac{|\sigma|}{h_{\sigma}}\left(v_{\sigma}-v_{K}\right) \varphi_{K} \\
& -\gamma\left[\sum_{K \in \mathcal{M}} \sum_{\sigma \in \mathcal{E}_{K} \& \sigma \subset \Gamma_{0}}|\sigma| \hat{v}_{K} \varphi_{K}-\sum_{K \in \mathcal{M}} \sum_{\sigma \in \mathcal{E}_{K} \& \sigma \subset \Gamma_{1}}|\sigma| \hat{\varphi}_{K} v_{K}\right] \\
& +\sum_{K \in \mathcal{M}} \varphi_{K} \hat{f}_{K} \\
& \equiv-J_{1}-J_{2}+J_{3}-J_{4}-J_{5}+J_{6},
\end{aligned}
$$

where $\hat{\varphi}_{K}$ is the value $\varphi\left(\hat{x}_{K}\right)$ for the mirror image of $K$.

Since we have regular meshes and established in previous sections convergence properties for the sequence $\left\{v^{N}\right\}$, then (6.1) with $v^{N}, \hat{f}^{N}$ (defined by (5.1) for the mesh $\mathcal{M}^{N}$ ) instead $v, \hat{f}$ gives

$$
\begin{aligned}
& J_{1} \rightarrow \int_{\Omega} v_{0} \Delta \varphi d x \quad \text { as } N \rightarrow \infty, \quad J_{2} \rightarrow \gamma \int_{\Omega} \varphi_{x_{3}} v_{0} d x \quad \text { as } N \rightarrow \infty, \\
& J_{3} \rightarrow \int_{\partial \Omega} \operatorname{tr} v_{0} \frac{\partial \varphi}{\partial n} d S \quad \text { as } N \rightarrow \infty \\
& J_{4} \rightarrow \int_{\Gamma}\left(G\left(v_{0}+u_{*}\right)-\frac{\partial u_{*}}{\partial n}\right) \varphi d S \quad \text { as } N \rightarrow \infty \\
& J_{5} \rightarrow \gamma \int_{\Gamma_{1}} \operatorname{tr} v_{0} \varphi d S \quad \text { as } N \rightarrow \infty \\
& J_{6} \rightarrow-\int_{\Omega} u_{*} \Delta \varphi d x+\int_{\partial \Omega} u_{*} \frac{\partial \varphi}{\partial n} d S-\int_{\Gamma} \frac{\partial u_{*}}{\partial n} \varphi d S \quad \text { as } N \rightarrow \infty
\end{aligned}
$$


This way

$$
\begin{aligned}
& -\int_{\Omega}\left(v_{0}+u_{*}\right) \triangle \varphi d x-\gamma \int_{\Omega}\left(v_{0}+u_{*}\right) \varphi_{x_{3}} d x+\int_{\partial \Omega}\left(v_{0}+u_{*}\right) \frac{\partial \varphi}{\partial n} d S \\
& -\int_{\Gamma} G\left(v_{0}+u_{*}\right) \varphi d S+\gamma \int_{\Gamma_{1}} v_{0} \varphi d S=0
\end{aligned}
$$

for all $\varphi \in C^{2}\left(\mathbb{R}^{3}\right)$, which are zero in a neighborhood of $\Gamma_{0}$.

Since $v_{0} \in W_{2}^{1}(\Omega), u_{*} \in C^{2}\left(\mathbb{R}^{3}\right), u_{*}$ does not depend on $x_{3}$ and $v_{0}=0$ on $\Gamma_{0}$, then

$$
\begin{aligned}
& \int_{\Omega}\left[\left\langle\nabla\left(v_{0}+u_{*}\right), \nabla \varphi\right\rangle+\gamma\left(v_{0}+u_{*}\right)_{x_{3}} \varphi\right] d x \\
& \quad-\kappa \int_{\Gamma}\left\{\psi\left(v_{0}+u_{*}\right)-H\left[\psi\left(v_{0}+u_{*}\right)\right]\right\} \varphi d S+\int_{\Gamma} g \varphi d S=0
\end{aligned}
$$

for all $\varphi \in W_{2}^{1}(\Omega)$ with $\varphi=0$ on $\Gamma_{0}$.

Therefore, $v_{0}+u_{*}$ is the solution of our initial problem $(2.2)$.

By construction, for mesh $\mathcal{M}^{N}$ the function $w^{N}:=v^{N}+\bar{u}^{N}\left(\bar{u}^{N}\right.$ corresponds to $u_{*}$ for the mesh $\mathcal{M}^{N}$ ) is the solution for the family of equations (2.4). This way, we have the following result.

Theorem 3. Let the hypotheses $H_{1}-H_{3}$ hold and let $\left\{\mathcal{M}^{N}\right\}$ be a sequence of regular rectangular meshes, for which the size $d_{\mathcal{M}^{N}} \rightarrow 0$ as $N \rightarrow \infty$.

Then the discretized equations (2.4) of the finite volume method have unique solutions $w^{N}$ for every $N$ and the sequence $\left\{w^{N}\right\}$ converges strongly in $L_{2}(\Omega)$ and $L_{2}(\partial \Omega)$ to the solution $u$ of the continuous boundary value problem (2.2).

\section{References}

[1] K. Birgelis. Optimal control in models with conductive-radiative heat transfer. Math. Model. Anal., 8(1):1-12, 2003.

[2] K. Birgelis. Sensitivity analysis for an optimal control problem of heat transfer. University of Latvia, Riga, 2007. (PhD Thesis)

[3] K. Birgelis and U. Raitums. Strictly convergent algorithm for an elliptic equation with nonlocal and nonlinear boundary conditions. Math. Model. Anal., 17(1):128-139, 2012. http://dx.doi.org/10.3846/13926292.2012.647100.

[4] A. Buikis and A.D. Fitt. A mathematical model for the heat treatment of glass fabric sheets. IMA J. Math. Appl. Bus. Ind., 10(1):55-86, 1999. http://dx.doi.org/10.1093/imaman/10.1.55.

[5] R. Čiegis, G. Jankevičiūtè and O. Suboč. Numerical simulation of the heat conduction in composite materials. Math. Model. Anal., 15(1):9-22, 2010. http://dx.doi.org/10.3846/1392-6292.2010.15.9-22.

[6] R. Eymard, T. Gallouet and R. Herbin. Finite Volume Methods. University of Wroclaw, 2010.

[7] T. Gallouet, R. Herbin and M.H. Vignal. Error estimates on the approximate finite volume solution of convection diffusion equation with general boundary conditions. SIAM J. Numer. Anal., 37(6):1935-1972, 2000. http://dx.doi.org/10.1137/S0036142999351388. 
[8] M. Laitinen and T. Tiihonen. Conductive-radiative heat transfer in grey materials. Q. Appl. Math., 59(4):737-768, 2001.

[9] C. Mayer, P. Philip and F. Tröltzsch. Optimal control of a semilinear PDE with nonlocal radiation interface conditions. SIAM J. Control Optim., 45:699-721, 2006. http://dx.doi.org/10.1137/040617753.

[10] I.M. Stein. Singular Integrals and Differentiability Properties of Functions. Princeton University Press, Princeton, 1970. 